\title{
Formulation and Analysis of Horizontal Mergers Among Oligopolistic Firms with Insights into the Merger Paradox: A Supply Chain Network Perspective
}

\author{
Anna Nagurney \\ Department of Finance and Operations Management \\ Isenberg School of Management \\ University of Massachusetts \\ Amherst, Massachusetts 01003
}

August 2008; revised January and February 2009

Computational Management Science (2010), 7, 377-401.

\begin{abstract}
In this paper, we consider oligopolistic firms with supply chain networks who are involved in the production, storage, and distribution of a homogeneous product to demand markets and explore what has become known in the literature as the "merger paradox." We present the oligopolistic supply chain network equilibrium model associated with the competing firms before the horizontal mergers and also develop the supply chain network optimization model post the complete merger. In addition, we develop the model in which only a subset of the firms in the industry merge. The governing concept of the competing firms is that of Cournot-Nash equilibrium. We utilize finite-dimensional variational inequality theory for the formulation, analysis, and solution of both the pre and the post-merger supply chain network problems. We provide numerical examples for which we compute the total costs, the total revenues, as well as the profits obtained for the firms pre and post the mergers for a variety of distinct oligopoly problems. The generality of the network models and the flexibility of the computational approach, which yields closed form expressions for the product flows at each iteration, allows us to obtain deeper insights into the merger paradox.
\end{abstract}

This paper may be viewed as a contribution to the marriage of frameworks in operations research/management science and computational economics.

Keywords: oligopolies, supply chains, horizontal mergers, game theory, Nash equilibria, network economics, variational inequalities, merger paradox 


\section{Introduction}

In this paper, we consider the formulation, analysis, and solution of horizontal mergers between/among oligopolistic firms that are involved in the production of a homogeneous product and compete noncooperatively in a Cournot (1838) framework. Examples of some oligopolies include oil, beer, automobile manufacturing companies, airlines, and certain financial institutions in the United States, supermarket chains in the United Kingdom, as well as media outlets in Australia. Examples of recent mergers of oligopolistic firms include: the merger of Wells Fargo \& Co. and Wachovia Corp. in financial services (cf. Phoenix Business Journal (2009)), the merger of the airlines Delta and Northwest (Global News Wire (2008)), the merger of Anheuser-Busch and InBev in the beer/beverage industry (TradingMarkets.com (2008)) and that of Molson and Coors (Beverage World (2007)) as well as the merger of Exxon and Mobil in the oil industry (see CNNMoney.com (1999)).

The topic of mergers in an oligopolistic setting has been of major interest in economics and a subject of much discussion. Some notable papers include those by Salant, Switzer, and Reynolds (1983), Perry and Porter (1985), and Farrell and Shapiro (1990). However, much of the economics literature on this topic is limited to linear cost and demand structures. For a survey of analytical perspectives to mergers (and acquisitions), see Meschi (1997).

Interestingly, the topic of mergers (and acquisitions) (M\&As) has also been researched more recently by operations researchers/management scientists and, specifically, in the context of supply chains (cf. Gupta and Gerchak (2002)). In particular, Langabeer and Seifert (2003) showed a direct correlation between the level of success of the merged companies and how effectively the supply chains of the merged companies are integrated. As noted by Soylu et al. (2006), who developed a mixed integer linear programming model (see also Xu (2007)) of energy supply chains, more and more companies now realize the strategic importance of controlling the supply chain as a whole (see, also, Brown et al. (2001) for a specific corporate example). Furthermore, Min and Zhou (2002) emphasized the need to analyze the synergy obtained through both inter-functional and inter-organizational integration. Hakkinen et al. (2004) described the integration of logistics after M\&As with a review of the literature and concluded that operational issues, in general, and logistics issues, specifically, have received insufficient attention; see also Herd, Saksena, and Steger (2005). 
In this paper, we tackle the problem of horizontal mergers of firms engaged in oligopolistic competition but from a supply chain network perspective. We believe that such a perspective provides a powerful graphical means by which to visualize and study horizontal mergers between/among firms and in which to explore what is known in the literature as the "merger paradox." According to Pepall, Richards, and Norman (1999), "What may be surprising to you is that it is, in fact, quite difficult to construct a simple economic model in which there are sizable profitability gains for the firms participating in a horizontal merger that is not a merger to monopoly." Salant, Switzer, and Reynolds (1983) earlier emphasized that, in quantity-setting games, as we consider here, it is not usually advantageous for the merging firms unless the merger includes the vast majority of the firms, in particular, $80 \%$ or more (see also Creane and Davidson (2004)). In reality, however, mergers of only two firms in an industry, and not mergers (almost) to a monopoly (especially given antitrust laws) are more typical in occurrence and frequency (see also Ghosal and Stennek (2007)).

According to Kusstatscher and Cooper (2005) there have been five major waves of Merger and Acquisition activity:

The First Wave: 1898 - 1902: this wave consisted of an increase in horizontal mergers that resulted in many U.S. industrial groups;

The Second Wave: 1926 - 1939: involved many public utilities;

The Third Wave: 1969 - 1973: had as its driving force diversification;

The Fourth Wave: 1983 - 1986: had as its goal efficiency;

The Fifth Wave: 1997 until the early years of the 21st century: had as its motto globalization.

In the present uncertain economic and financial climate it is practically imperative to quantitatively assess a priori the potential cost savings associated with a proposed merger or acquisition. Towards that end, we depict each firm as a network of its economic activities of production, storage, and distribution to demand markets. We assume that the firms pre and post the merger produce a homogeneous good in a noncooperative manner. We identify the network structure of the merged firms which allows one to associate costs with the new links. 
Both the pre and the post-merger models are formulated as variational inequality problems (cf. Nagurney (1993)). Some of the history of the evolution of network models of firms and numerous network-based economic models can also be found in Nagurney (1993)).

We emphasize that although the presentation and the analysis are done from a supply chain perspective the concepts are even broader - for example, they may be applied to the integration of firms in a variety of network industries from telecommunications, transportation (railways, in addition to airlines), financial services, to energy, including electric power. Indeed, the originality of our approach lies in the conceptualization of the firms or organizations of interest as networks and their integration through mergers or acquisitions being formed through the addition of appropriate new links (with associated costs). Flows on the networks prior or post the integration correspond, depending upon the particular application, to products, people, messages, energy in a variety of forms, financial transactions, and so on. Of course, in other network economic settings, the "manufacturers" may correspond to producers of services, knowledge, etc., as appropriate, and the nodes and links that abstract the firms and their activities would be re(defined) accordingly as would the link costs and network flows.

Recently, a variety of supply chain network equilibrium models, initiated by Nagurney, Dong, and Zhang (2002) have been developed, which focus on competition among decisionmakers (such as manufacturers, distributors, and retailers) at a tier of the supply chain but cooperation between tiers. The relationships of such supply chain network equilibrium problems to transportation network equilibrium problems, which are characterized by useroptimizing behavior have also been established (cf. Nagurney (2006a)). Zhang, Dong, and Nagurney (2003) and Zhang (2006), in turn, modeled competition among supply chains in a supply chain economy context, but did not consider explicit firms and did not formulate mergers. See the book by Nagurney (2006b) for a spectrum of supply chain network equilibrium models and applications.

The supply chain network models in this paper, in contrast to the ones noted immediately above, are more detailed, since they are at the firm level. In addition, to the best of our knowledge, the models in this paper are the first to capture the supply chain network aspects of mergers of oligopolistic firms and the associated total costs and profits. The supply chain 
network merger models of Nagurney (2009) assumed that there were only two firms involved in a potential merger and that each firm's demand markets were separate from those of the other. Moreover, these models assumed that the firms were not in competition and that the demands were inelastic for the product at the demand markets. A synergy measure, which captured the total cost reduction, if any, was then introduced and associated with the horizontal merger. In this paper, in contrast, we assume that the finite number of firms compete in an oligopolistic manner and that there are price functions associated with the product at each demand market. Moreover, we utilize the change in total costs and total profits as measures to identify whether or not the merger would make economic sense. Finally, we explore questions surrounding the merger paradox, which is not possible in the context of the Nagurney (2009) model, which considered mergers of only two cost-minimizing firms.

This paper is organized as follows. In Section 2, we develop the supply chain network models pre and post the mergers. We formulate both partial as well as complete merger models using a graphical, supply chain network formalism. The supply chain network problems are formulated as finite-dimensional variational inequalities. We provide alternative variational inequality formulations, which induce different computational procedures. We also highlight a special case.

In Section 3, we compute solutions to numerical examples for a wide variety of supply chain network structures of oligopolistic firms and evaluate the horizontal mergers, both partial and complete. We also explore questions regarding the merger paradox computationally, an approach which allows one to gain insights through numerical experimentation. We determine the total costs, the revenues, and the profits for the supply chain networks pre and post the mergers. For convenience of the reader, in the Appendix, we give the statement of the algorithm and its realizations for the network models. In addition, we derive some theoretical results.

In Section 4, we summarize the results in this paper and give suggestions for future research. 
Firm 1

Firm $I$

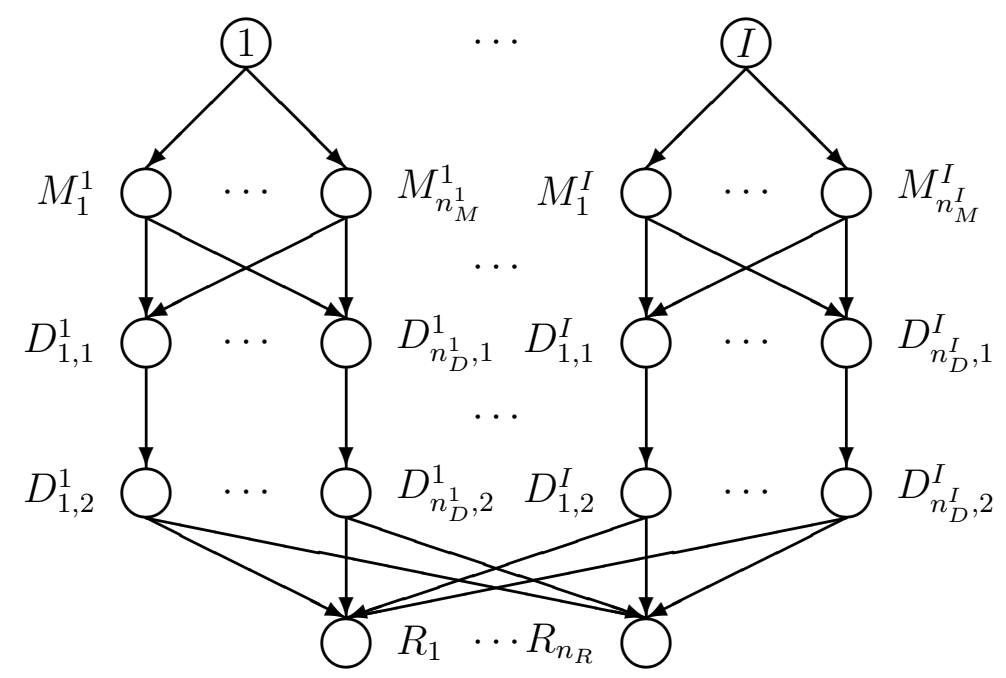

Figure 1: Supply Chains of the Oligopolistic Firms $i ; i=1, \ldots, I$ Prior to the Merger/Integration

\section{The Pre- and Post-Horizontal Merger Supply Chain Network Models}

In this Section, we describe the supply chain network models prior and post the horizontal mergers. We consider a finite number of firms, denoted by Firm 1, Firm 2, and so on, until Firm $I$, who are involved in the production, storage, and distribution of a homogeneous product and who compete noncooperatively in an oligopolistic manner.

In Section 2.1, we formulate the pre-merger oligopolistic supply chain network equilibrium problem and we consider this as the baseline case, Case 0. In Section 2.2, we formulate the post-merger model, corresponding to Case 1, in which all $I$ firms merge. In addition, in Section 2.3, we develop the model in which a subset of the $I$ firms merges, and we refer to this case as Case 2. The governing equilibrium/optimality conditions of all the models are formulated as variational inequality problems.

\subsection{The Pre-Merger Supply Chain Network Oligopoly Model}

We assume that each firm is represented as a network of its economic activities (cf. Figure 
1). Each firm $i ; i=1, \ldots, I$ has $n_{M}^{i}$ manufacturing facilities/plants; $n_{D}^{i}$ distribution centers, and serves the same $n_{R}$ retail outlets/demand markets. Let $L_{i}^{0}$ denote the set of directed links representing the economic activities associated with firm $i ; i=1, \ldots, I$. Let $L^{0} \equiv \cup_{i=1, I} L_{i}^{0}$. Let $G^{0}=\left[N^{0}, L^{0}\right]$ denote the graph consisting of the set of nodes $N^{0}$ and the set of links $L^{0}$ in Figure 1.

The links from the top-tiered nodes $i ; i=1, \ldots, I$ in Figure 1 are connected to the manufacturing nodes of the respective firm $i$, which are denoted, respectively, by: $M_{1}^{i}, \ldots, M_{n_{M}^{i}}^{i}$, and these links represent the manufacturing links. The links from the manufacturing nodes, in turn, are connected to the distribution center nodes of each firm $i ; i=1, \ldots, I$, which are denoted by $D_{1,1}^{i}, \ldots, D_{n_{D^{i}, 1}}^{i}$. These links correspond to the shipment links between the manufacturing plants and the distribution centers where the product is stored. The links joining nodes $D_{1,1}^{i}, \ldots, D_{n_{D}^{i}, 1}^{i}$ with nodes $D_{1,2}^{i}, \ldots, D_{n_{D}^{i}, 2}^{i}$ for $i=1, \ldots, I$ correspond to the storage links. Finally, there are shipment links joining the nodes $D_{1,2}^{i}, \ldots, D_{n_{D}^{i}, 2}^{i}$ for $i=1, \ldots, I$ with the demand market nodes: $R_{1}, \ldots, R_{n_{R}}$.

We assume that associated with each link (cf. Figure 1) of the network corresponding to each firm $i ; i=1, \ldots, I$ is a total cost. We denote, without any loss in generality, the links by $a, b$, etc., and the total cost on a link $a$ by $\hat{c}_{a}$. Let $d_{R_{k}}$ denote the demand for the product at demand market $R_{k} ; k=1, \ldots, n_{R}$. Let $x_{p}$ denote the nonnegative flow of the product on path $p$ joining (origin) node $i$ with a (destination) demand market node. Then the following conservation of flow equations must hold:

$$
d_{R_{k}}=\sum_{p \in P_{R_{k}}^{0}} x_{p}, \quad k=1, \ldots, n_{R},
$$

where $P_{R_{k}}^{0}$ denotes the set of paths connecting the (origin) nodes $i ; i=1, \ldots, I$ with (destination) demand market $R_{k}$. In particular, we have that $P_{R_{k}}^{0}=\cup_{i=1, \ldots, I} P_{R_{k}^{i}}^{0}$, where $P_{R_{k}^{i}}^{0}$ denotes the set of paths from origin node $i$ to demand market $k$ as in Figure 1.

According to (1), the demand at each demand market must be equal to the sum of the product flows from all firms to that demand market.

We assume that there is a demand price function associated with the product at each demand market. We denote the demand price at demand market $R_{k}$ by $\rho_{R_{k}}$ and we assume, 
as given, the demand price functions:

$$
\rho_{R_{k}}=\rho_{R_{k}}\left(d_{R_{k}}\right), \quad k=1, \ldots, n_{R}
$$

We assume that the demand price functions are continuous, continuously differentiable, and monotone decreasing. Note that the consumers at each demand market are indifferent as to which firm produced the homogeneous product.

In addition, we let $f_{a}$ denote the flow of the product on link $a$. Hence, we must also have the following conservation of flow equations satisfied:

$$
f_{a}=\sum_{p \in P^{0}} x_{p} \delta_{a p}, \quad \forall a \in L^{0}
$$

where $\delta_{a p}=1$ if link $a$ is contained in path $p$ and $\delta_{a p}=0$, otherwise. Here $P^{0}$ denotes the set of all paths in Figure 1, that is, $P^{0}=\cup_{k=1, \ldots, n_{R}} P_{R_{k}}^{0}$. There are $n_{P^{0}}$ paths in the pre-merger network in Figure 1. Obviously, prior to any merger the paths associated with a given firm have no links in common with paths of any other firm. This changes when the horizontal mergers occur, in which case the number of paths and the sets of paths also change, as do the number of links and the sets of links, as we demonstrate in Sections 2.2 and 2.3. We use $P_{i}^{0}$ to denote the set of all paths from firm $i$ to all the demand markets for $i=1, \ldots, I$. There are $n_{P_{i}^{0}}$ paths from the firm $i$ node to the demand markets.

Of course, we also have that the path flows must be nonnegative, that is,

$$
x_{p} \geq 0, \quad \forall p \in P^{0} .
$$

The total cost on a link, be it a manufacturing/production link, a shipment/distribution link, or a storage link is assumed to be a function of the flow of the product on the link; see, for example, Nagurney (2009) and the references therein. We denote the total cost on link $a$ by $\hat{c}_{a}$ and we assume that:

$$
\hat{c}_{a}=\hat{c}_{a}\left(f_{a}\right), \quad \forall a \in L^{0} .
$$

We assume that the total cost on each link is convex and continuously differentiable. We assume the same for all links that are added post the mergers, as well. Such assumptions are standard in classical oligopoly theory (see, e.g., Gabay and Moulin (1980), Friedman (1982), 
Murphy, Sherali, and Soyster (1982), Tirole (1988) and the references therein). Also, such assumptions are reasonable with respect to the transportation and shipment links due to congestion (see Dafermos and Sparrow (1969)). Note that in Nagurney (2009) there were explicit capacities imposed on the links. Here we assume that the capacities are handled within the cost functions, if need be. Of course, a special case of total cost function (5) that satisfies the above assumptions is a linear, separable function, such that, for example, $\hat{c}_{a}=h_{a} f_{a}$ for $h_{a}$ nonnegative.

The profit function $u_{i}$ of firm $i ; i=1, \ldots, I$, is the difference between the firm's revenue and its total costs, that is,

$$
u_{i}=\sum_{k=1}^{n_{R}} \rho_{R_{k}}\left(d_{R_{k}}\right) \sum_{p \in P_{R_{k}^{0}}^{0}} x_{p}-\sum_{a \in L_{i}^{0}} \hat{c}_{a}\left(f_{a}\right) .
$$

In view of (1) and (3), and (2) and (5), we may write:

$$
u=u(x),
$$

where $x$ is the vector of all the path flows $\left\{x_{p}, p \in P^{0}\right\}$, and $u$ is the $I$-dimensional vector of the firms' profits.

We now consider the usual oligopolistic market mechanism in which the $I$ firms produce and distribute the product in a noncooperative manner, each one trying to maximize its own profit. We seek to determine a nonnegative path flow pattern $x$ for which the $I$ firms will be in a state of equilibrium as defined below.

\section{Definition 1: Pre-Merger Supply Chain Network Cournot-Nash Equilibrium}

A product flow pattern $x^{*} \in R_{+}^{n_{P 0}}$ is said to constitute a supply chain network Cournot-Nash equilibrium if for each firm $i ; i=1, \ldots, I$ :

$$
u_{i}\left(x_{i}^{*}, \hat{x}_{i}^{*}\right) \geq u_{i}\left(x_{i}, \hat{x}_{i}^{*}\right), \quad \forall x_{i} \in R_{+}^{n_{P_{i}^{0}}},
$$

where $x_{i} \equiv\left\{\left\{x_{p}\right\} \mid p \in P_{i}^{0}\right\}$ and $\hat{x}_{i}^{*} \equiv\left(x_{1}^{*}, \ldots, x_{i-1}^{*}, x_{i+1}^{*}, \ldots, x_{I}^{*}\right)$.

Note that, according to (8), a Cournot-Nash equilibrium has been established if no firm can increase its profits unilaterally. 
The variational inequality formulation of the Cournot-Nash (Cournot (1838), Nash (1950, 1951)) supply chain network equilibrium satisfying Definition 1 is given in the following theorem, along with two variants.

\section{Theorem 1}

Assume that for each firm $i ; i=1, \ldots, I$, the profit function $u_{i}(x)$ is concave with respect to the variables $x_{p} ; p \in P_{i}^{0}$, and is continuously differentiable. Then $x^{*} \in R_{+}^{n_{P^{0}}}$ is a pre-merger supply chain network Cournot-Nash equilibrium if and only if it satisfies the variational inequality:

$$
-\sum_{i=1}^{I} \sum_{p \in P_{i}^{0}} \frac{\partial u_{i}\left(x^{*}\right)}{\partial x_{p}} \times\left(x_{p}-x_{p}^{*}\right) \geq 0, \quad \forall x \in R_{+}^{n_{P^{0}}} .
$$

Upon using (1), (3), (2), and (5), variational inequality (9) may be reexpressed as: determine $\left(f^{*}, x^{*}, d^{*}\right) \in \mathcal{K}^{0 a}$, such that

$$
\begin{gathered}
\sum_{a \in L^{0}} \frac{\partial \hat{c}_{a}\left(f_{a}^{*}\right)}{\partial f_{a}} \times\left(f_{a}-f_{a}^{*}\right)-\sum_{k=1}^{n_{R}} \rho_{R_{k}}\left(d_{R_{k}}^{*}\right) \times\left(d_{R_{k}}-d_{R_{k}}^{*}\right) \\
-\sum_{i=1}^{I} \sum_{p \in P_{i}^{0}}\left[\sum_{k=1}^{n_{R}} \frac{\partial \rho_{R_{k}}\left(d_{R_{k}}^{*}\right)}{\partial d_{R_{k}}} \sum_{p \in P_{R_{k}^{i}}^{0}} x_{p}^{*}\right] \times\left(x_{p}-x_{p}^{*}\right) \geq 0, \quad \forall(f, x, d) \in \mathcal{K}^{0 a},
\end{gathered}
$$

where $\mathcal{K}^{0 a} \equiv\left\{(f, x, d) \mid x \in R_{+}^{n_{P^{0}}}\right.$ and(1) and(3) hold $\}$ or, equivalently, due to (1) and (3): determine $x^{*} \in \mathcal{K}^{0 b}$ satisfying:

$\sum_{i=1}^{I} \sum_{k=1}^{n_{R}} \sum_{p \in P_{R_{k}^{i}}^{0}}\left[\frac{\partial \hat{C}_{p}\left(x^{*}\right)}{\partial x_{p}}-\rho_{R_{k}}\left(\sum_{p \in P_{R_{k}}^{0}} x_{p}^{*}\right)-\frac{\partial \rho_{R_{k}}\left(\sum_{p \in P_{R_{k}}^{0}} x_{p}^{*}\right)}{\partial d_{R_{k}}} \sum_{p \in P_{R_{k}^{0}}^{0}} x_{p}^{*}\right] \times\left[x_{p}-x_{p}^{*}\right] \geq 0, \forall x \in \mathcal{K}^{0 b}$,

where $\mathcal{K}^{0 b} \equiv\left\{x \mid x \in R_{+}^{n_{P^{0}}}\right\}$ and $\frac{\partial \hat{C}_{p}(x)}{\partial x_{p}} \equiv \sum_{a \in L^{0}} \frac{\partial \hat{c}_{a}\left(f_{a}\right)}{\partial f_{a}} \delta_{a p}$.

Proof: Follows directly from Gabay and Moulin (1982) and Dafermos and Nagurney (1987). Here we have also utilized the fact that the demand price functions (2) can be reexpressed in light of (1) directly as a function of path flows.

It is interesting to relate this model to the classical Cournot (1838) oligopoly model, which has been formulated as a variational inequality problem by Gabay and Moulin (1982) and 


\section{Firm $1 \quad$ Firm $I$}

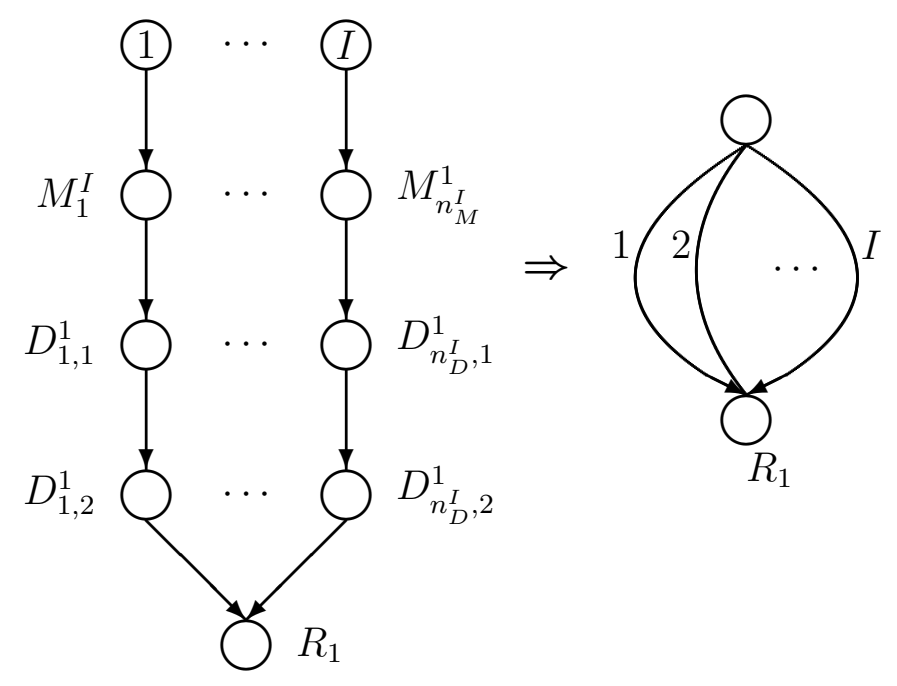

Figure 2: Network Structure of the Classical Oligopoly

has been studied by both economists and operations researchers (cf. Murphy, Sherali, and Soyster (1982), Flam and Ben-Israel (1990), Nagurney (1993), and the references therein). Indeed, we have the following Corollary, the proof of which is immediate.

\section{Corollary 1}

Assume that there is a single manufacturing plant associated with each firm in the above model, and a single distribution center. Assume also that there is a single demand market. Then, if the storage and distribution cost functions are all identically equal to zero the above model collapses to the classical oligopoly model. Furthermore, if $I=2$, one then obtains the classical duopoly model.

The network structure for the classical oligopoly problem (see also Nagurney (1993)) is depicted in Figure 2. 


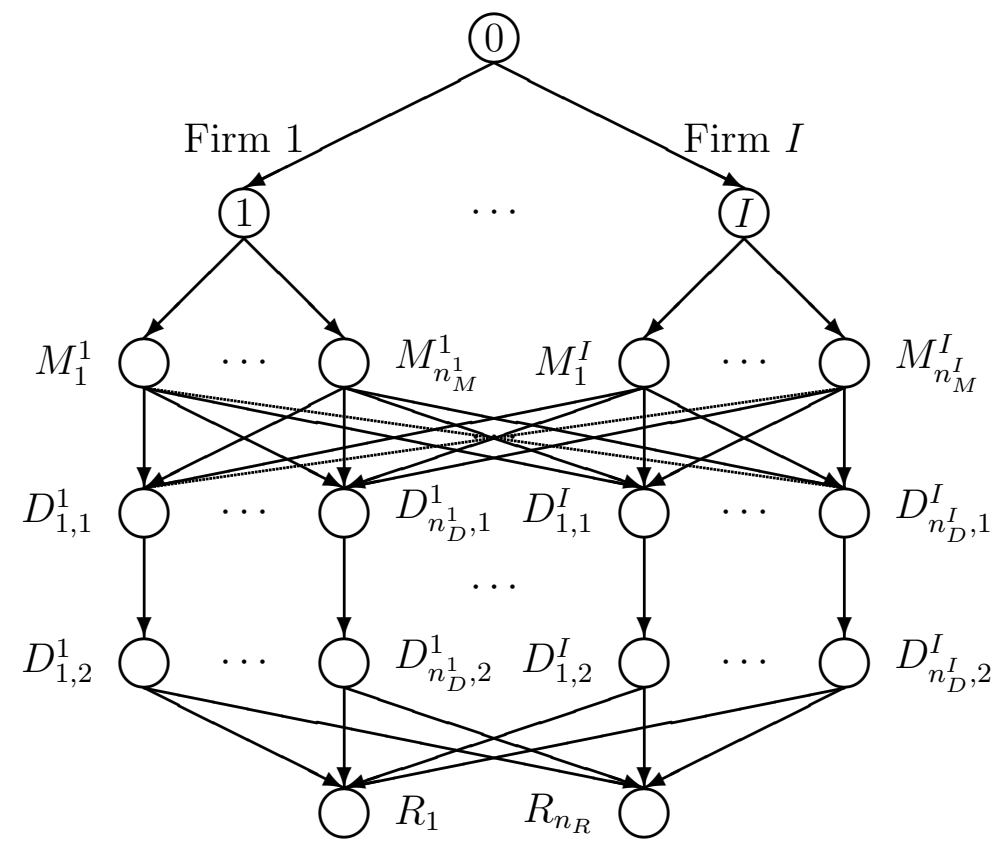

Figure 3: The Integrated Supply Chain Network Representing the Merger of All the Firms

\subsection{The Complete Horizontal Merger Supply Chain Network Model}

In this Section, we consider the merger of all the firms $i ; i=1, \ldots, I$. Specifically, we retain the nodes and links associated with network $G^{0}$ depicted in Figure 1 but now the additional links connecting the manufacturing plants of each firm to the distribution centers of all the other original firms are added. We refer to the network underlying this merger as $G^{1}=\left[N^{1}, L^{1}\right]$ where $N^{1}=N^{0} \cup$ node 0 and $L^{1}$ is the set of all links as in Figure 3 . We associate with the new links total cost functions as in (5) but now for all $a \in L^{1}$. The demand price functions remain as in (2).

Let $x_{p}$ now denote the flow of the product on path $p$ joining (origin) node 0 with a (destination) demand market node. Then the following conservation of flow equations must hold:

$$
d_{R_{k}}=\sum_{p \in P_{R_{k}}^{1}} x_{p}, \quad k=1, \ldots, n_{R},
$$

where $P_{R_{k}}^{1}$ denotes the set of paths connecting node 0 with demand market node $R_{k}$ in Figure 3. Due to the merger of all the firms, the product can be stored at any of the distribution 
centers and shipped then to the demand markets.

In addition, as before, we let $f_{a}$ denote the flow of the product on link $a$. Hence, we must now have the following conservation of flow equations satisfied:

$$
f_{a}=\sum_{p \in P^{1}} x_{p} \delta_{a p}, \quad \forall a \in L^{1}
$$

where $P^{1}$ is the set of all paths joining node 0 with the demand market nodes in Figure 3.

Of course, we also have that the path flows must be nonnegative, that is,

$$
x_{p} \geq 0, \quad \forall p \in P^{1} .
$$

Since the firms are now merged into a single firm and, hence, they are no longer in competition, the single new firm has the following associated profit function, denoted by $U$, and expressed as:

$$
U=\sum_{k=1}^{n_{R}} \rho_{R_{k}}\left(d_{R_{k}}\right) \sum_{p \in P_{R_{k}}^{1}} x_{p}-\sum_{a \in L^{1}} \hat{c}_{a}\left(f_{a}\right) .
$$

In view of (11) and (12), and that we retain the demand price functions as in (2) and the total cost functions on all the links in Figure 3 as in (5), we may write the optimization problem faced by the new firm, which we assume seeks to maximize its profits, as:

$$
\text { Maximize }_{x \in R_{+}^{n_{P}}} \quad U=U(x)
$$

where $n_{P^{1}}$ denotes the number of paths joining node 0 to the demand markets in Figure 3 .

The following theorem is then immediate (see also Bazaraa, Sherali, and Shetty (1993)):

\section{Theorem 2}

Under the assumption that the profit function $U$ is concave with respect to each variable $x_{p}$, $p \in P^{1}$, and is continuously differentiable, the optimal solution to problem (15) is equivalent to the solution of the variational inequality problem: determine $x^{*} \in R_{+}^{n_{P}{ }^{1}}$ such that

$$
-\sum_{p \in P^{1}} \frac{\partial U\left(x^{*}\right)}{\partial x_{p}} \times\left(x_{p}-x_{p}^{*}\right) \geq 0, \quad \forall x \in R_{+}^{n_{P^{1}}},
$$


which, by taking into consideration (11), (12), (2), and (5) can be expressed as: determine $\left(f^{*}, d^{*}\right) \in \mathcal{K}^{1 a}$ satisfying:

$\sum_{a \in L^{1}} \frac{\partial \hat{c}_{a}\left(f_{a}^{*}\right)}{\partial f_{a}} \times\left(f_{a}-f_{a}^{*}\right)-\sum_{k=1}^{n_{R}}\left[\rho_{R_{k}}\left(d_{R_{k}}^{*}\right)+\frac{\partial \rho_{k}\left(d_{R_{k}}^{*}\right)}{\partial d_{R_{k}}} d_{R_{k}^{*}}\right] \times\left(d_{R_{k}}-d_{R_{k}}^{*}\right) \geq 0, \quad \forall(f, d) \in \mathcal{K}^{1 a}$,

where $\mathcal{K}^{1 a} \equiv\left\{(f, d) \mid\right.$ such that there exists $x \in R_{+}^{n_{P^{1}}}$ and (11) and (12)hold $\}$.

Equivalently, (17a) may be expressed, due to (11) and (12), as: determine $x^{*} \in \mathcal{K}^{1 b}$ satisfying:

$\left.\sum_{k=1}^{n_{R}} \sum_{p \in P_{R_{k}}^{1}}\left[\frac{\partial \hat{C}_{p}\left(x^{*}\right)}{\partial x_{p}}-\rho_{R_{k}}\left(\sum_{p \in P_{R_{k}}^{1}} x_{p}^{*}\right)-\frac{\partial \rho_{k}\left(\sum_{p \in P_{R_{k}}^{1}} x_{p}^{*}\right)}{\partial d_{R_{k}}} \sum_{p \in P_{R_{k}}^{1}} x_{p}^{*}\right)\right] \times\left(x_{p}-x_{p}^{*}\right) \geq 0, \quad \forall x \in \mathcal{K}^{1 b}$,

where $\mathcal{K}^{1 b} \equiv\left\{x \mid x \in R_{+}^{n_{P^{1}}}\right\}$ and here $\frac{\partial \hat{C}_{p}\left(x^{*}\right)}{\partial x_{p}} \equiv \sum_{a \in L^{1}} \frac{\partial \hat{c}_{a}\left(f_{a}\right)}{\partial f_{a}} \delta_{a p}$. 


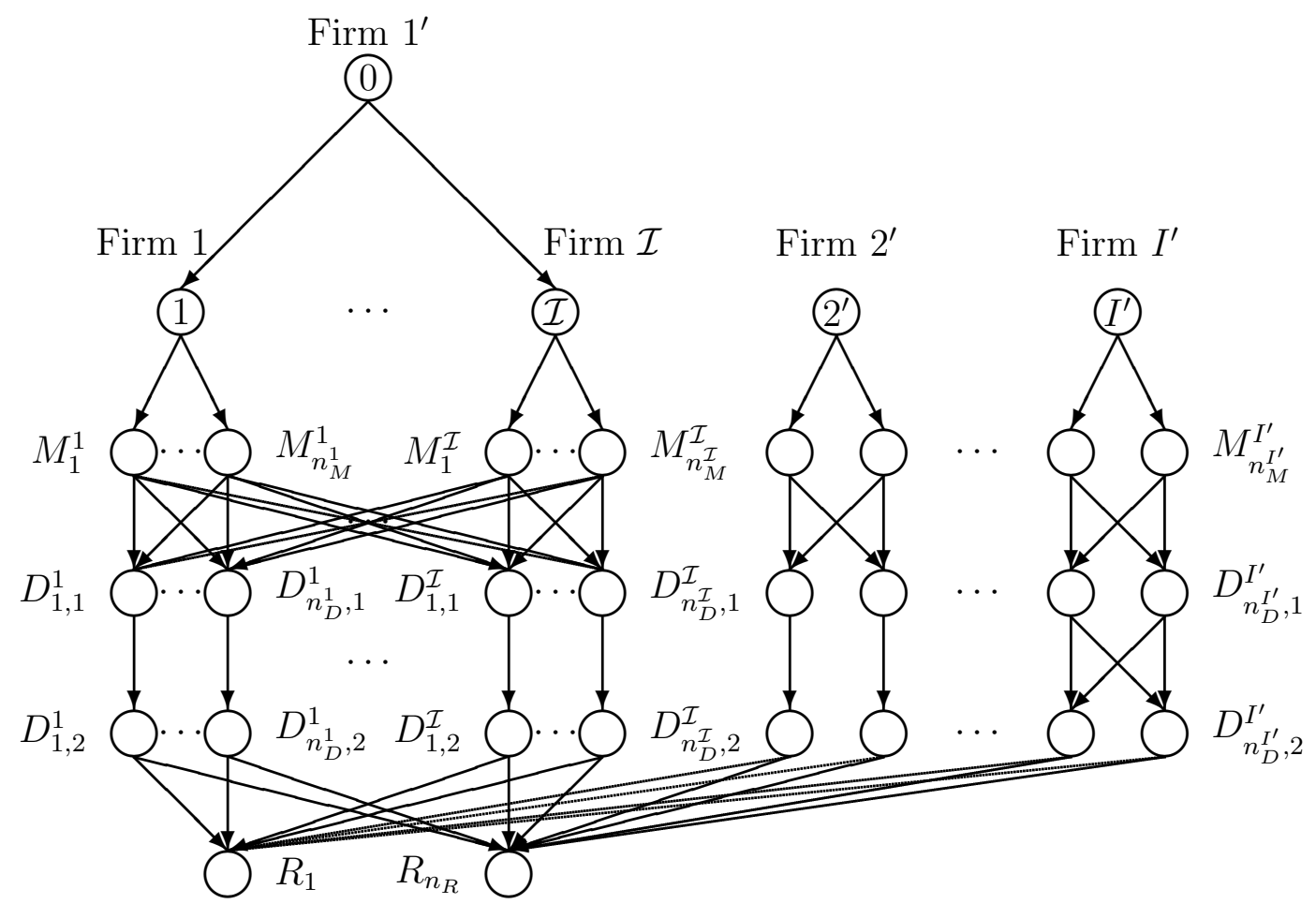

Figure 4: The Supply Chain Network Post the Partial Merger

\subsection{The Partial Horizontal Merger Supply Chain Network Model}

For completeness, we now present the partial merger model. This model is interesting and relevant, since not all firms in an industry necessarily need to merge when a merger occurs. Such a model also allows one to evaluate the effect of the merger on total costs and profits of firms not associated with the merger. Moreover, it allows on to explore questions regarding the merger paradox.

We assume that, for simplicity, the first $\mathcal{I}$ firms merge, where $\mathcal{I}<I$. The supply chain network structure of such a merger is depicted in Figure 4 . We refer to the network for the partial merger as $G^{2}=\left[N^{2}, L^{2}\right]$ where $N^{2}=N^{1}$ but $L^{2}$ denotes all the links in Figure 4. Clearly, the sets of paths and the conservation of flow equations for this model need to be stated. 
We define $P_{R_{k}^{i}}^{2}$ as the set of paths joining origin node $i$ with demand market $R_{k}$, where $i=1^{\prime}, 2^{\prime}, \ldots, I^{\prime}=I-\mathcal{I}+1$.

Let $x_{p}$ now denote the nonnegative flow on a path joining (origin) node $i$ with a demand market node in Figure 4. Then the following conservation of flow equations must now be satisfied in the partial merger case:

$$
\sum_{p \in P_{R_{k}}^{2}} x_{p}=d_{R_{k}}, \quad k=1, \ldots, n_{R}
$$

where $P_{R_{k}}^{2}=\cup_{i=1^{\prime}, \ldots, I^{\prime}} P_{R_{k}^{i}}^{2}$. We let $P_{i}^{2}$ denote the set of all paths emanating from node $i$ to the demand markets for $i=1^{\prime}, \ldots, I^{\prime}$.

The demand price functions remain as in Cases 0 and 1. The link conservation of flow equations now take the form:

$$
f_{a}=\sum_{p \in P^{2}} x_{p} \delta_{a p}, \quad \forall a \in L^{2}
$$

where $P^{2}=\cup_{k=1, \ldots, r_{R}} P_{R_{k}}^{2}$.

Of course, as in the preceding two models, we must have that the path flows are nonnegative, that is,

$$
x_{p} \geq 0, \quad \forall p \in P^{2} .
$$

Again, we assume that the new links that correspond to the partial merger have total cost functions associated with them; hence,

$$
\hat{c}_{a}=\hat{c}_{a}\left(f_{a}\right), \quad \forall a \in L^{2},
$$

and the new total cost functions on the new links have properties corresponding to those as in the original links. Of course, we retain the original total cost functions on the original links.

The profit now for the firms, including the new firm resulting from the merger of the first $\mathcal{I}$ firms and referred to as $1^{\prime}$, with the firms renumbered as: $i=1^{\prime}, \ldots, I^{\prime}$ can be expressed as:

$$
u_{i}=\sum_{k=1}^{n_{R}} \rho_{R_{k}}\left(d_{R_{k}}\right) \sum_{p \in P_{R_{k}^{2}}^{2}} x_{p}-\sum_{a \in L_{i}^{2}} \hat{c}_{a}\left(f_{a}\right),
$$


where $L_{i}^{2}$ denotes that subset of links in $L^{2}$ corresponding to firm $i ; i=1^{\prime}, \ldots, I^{\prime}$.

We can now adapt Definition 1 to this particular partial merger setting, in which firms $1^{\prime}, \ldots, I^{\prime}$ compete with one another in a Cournot-Nash setting until the equilibrium is attained. We impose the same assumptions on the utility functions here as were imposed on the utility functions in Theorem 1.

\section{Definition 2: Partial Merger Supply Chain Cournot-Nash Equilibrium}

A product flow pattern $x^{*} \in R_{+}^{n_{P^{2}}}$ is said to constitute a supply chain network Cournot-Nash equilibrium for the partial merger if for each firm $i ; i=1^{\prime}, \ldots, I^{\prime}$ :

$$
u_{i}\left(x_{i}^{*}, \hat{x}_{i}^{*}\right) \geq u_{i}\left(x_{i}, \hat{x}_{i}^{*}\right), \quad \forall x_{i} \in R_{+}^{n_{P_{i}^{2}}},
$$

where now, w.l.o.g. $x_{i} \equiv\left\{\left\{x_{p}\right\} \mid p \in P_{i}^{2}\right\}$ and $\hat{x}_{i}^{*} \equiv\left(x_{1^{\prime}}^{*}, \ldots, x_{i-1}^{*}, x_{i+1}^{*}, \ldots, x_{I^{\prime}}^{*}\right)$.

\section{Theorem 3}

Assume that for each firm $i ; i=1^{\prime}, \ldots, I^{\prime}$, the profit function $u_{i}(x)$ given by (22) is concave with respect to the variables $x_{p} ; p \in P_{i}^{2}$, and is continuously differentiable. Then $x^{*} \in R_{+}^{n_{P^{2}}}$ is a partial merger supply chain Cournot-Nash equilibrium according to Definition 2 if and only if it satisfies the variational inequality:

$$
-\sum_{i=1}^{I^{\prime}} \sum_{p \in P_{i}^{2}} \frac{\partial u_{i}\left(x^{*}\right)}{\partial x_{p}} \times\left(x_{p}-x_{p}^{*}\right) \geq 0, \quad \forall x \in R_{+}^{n_{P^{2}}} .
$$

Upon using (18), (19), (2), and (21), variational inequality (24) may be reexpressed as: determine $\left(f^{*}, x^{*}, d^{*}\right) \in \mathcal{K}^{2 a}$, such that

$$
\begin{gathered}
\sum_{a \in L^{2}} \frac{\partial \hat{c}_{a}\left(f_{a}^{*}\right)}{\partial f_{a}} \times\left(f_{a}-f_{a}^{*}\right)-\sum_{k=1}^{n_{R}} \rho_{R_{k}}\left(d_{R_{k}}^{*}\right) \times\left(d_{R_{k}}-d_{R_{k}}^{*}\right) \\
-\sum_{i=1}^{I^{\prime}} \sum_{p \in P_{i}^{2}}\left[\sum_{k=1}^{n_{R}} \frac{\partial \rho_{R_{k}}\left(d_{R_{k}}^{*}\right)}{\partial d_{R_{k}}} \sum_{p \in P_{R_{k}^{i}}^{2}} x_{p}^{*}\right] \times\left(x_{p}-x_{p}^{*}\right) \geq 0, \quad \forall(f, x, d) \in \mathcal{K}^{2 a},
\end{gathered}
$$

where $\mathcal{K}^{2 a} \equiv\left\{(f, x, d) \mid x \in R_{+}^{n_{P^{2}}}\right.$ and(18) and(19) hold $\}$ or, equivalently, in view of (18) and 
(19), determine $x^{*} \in \mathcal{K}^{2 b}$ satisfying:

$\sum_{i=1}^{I^{\prime}} \sum_{k=1}^{n_{R}} \sum_{p \in P_{R_{k}^{i}}^{0}}\left[\frac{\partial \hat{C}_{p}\left(x^{*}\right)}{\partial x_{p}}-\rho_{R_{k}}\left(\sum_{p \in P_{R_{k}^{2}}} x_{p}^{*}\right)-\frac{\partial \rho_{R_{k}}\left(\sum_{p \in P_{R_{k}^{2}}} x_{p}^{*}\right)}{\partial d_{R_{k}}} \sum_{p \in P_{R_{k}^{2}}^{2}} x_{p}^{*}\right] \times\left[x_{p}-x_{p}^{*}\right] \geq 0, \quad \forall x \in \mathcal{K}^{2 b}$,

where $\mathcal{K}^{2 b} \equiv\left\{x \mid x \in R_{+}^{n_{P^{2}}}\right\}$ and here $\frac{\partial \hat{C}_{p}(x)}{\partial x_{p}} \equiv \sum_{a \in L^{2}} \frac{\partial \hat{c}_{a}\left(f_{a}\right)}{\partial f_{a}} \delta_{a p}$.

For existence results for both classical and spatial oligopoly problems, see Nagurney and Zhang (1996b). The results therein can be adapted to this supply chain network setting.

\section{Numerical Examples}

In this Section, we present three sets of numerical oligopoly examples of increasing complexity. In Set 1, reported in Section 3.1, we present duopoly examples in which each firm has a single manufacturing plant and a single distribution center. There is assumed to be a single demand market. In Set 2, reported in Section 3.2, we consider oligopoly problems consisting of three firms, each with its individual manufacturing plant and distribution center, and a single demand market, and we compute solutions to both the partial merger and the complete merger problems. Finally, in Section 3.3, we report Set 3 numerical examples, in which we compute solutions to the mergers (both partial and complete) of more complex supply chain network structures than those reported in Sections 3.1 and 3.2.

We implemented the Euler method, as outlined in the Appendix, for each of the particular supply chain network problems, pre and post the mergers. The codes were implemented in FORTRAN and the system used for the computations was a Unix system at the University of Massachusetts Amherst. The convergence tolerance was: $\left|X^{\tau+1}-X^{\tau}\right| \leq .001$ for all the examples. The sequence $\left\{a_{\tau}\right\}$ used (cf. (A.1)) was: .1 $\left\{1, \frac{1}{2}, \frac{1}{2}, \frac{1}{3}, \frac{1}{3}, \frac{1}{3}, \ldots,\right\}$.

\subsection{Set 1}

The supply chain network structure for Problem Set 1 is given in Figure 5 .

\section{Example 1.1 Data and Solution}

This example is a pre-merger problem and it is a duopoly. It is used as a baseline for the 


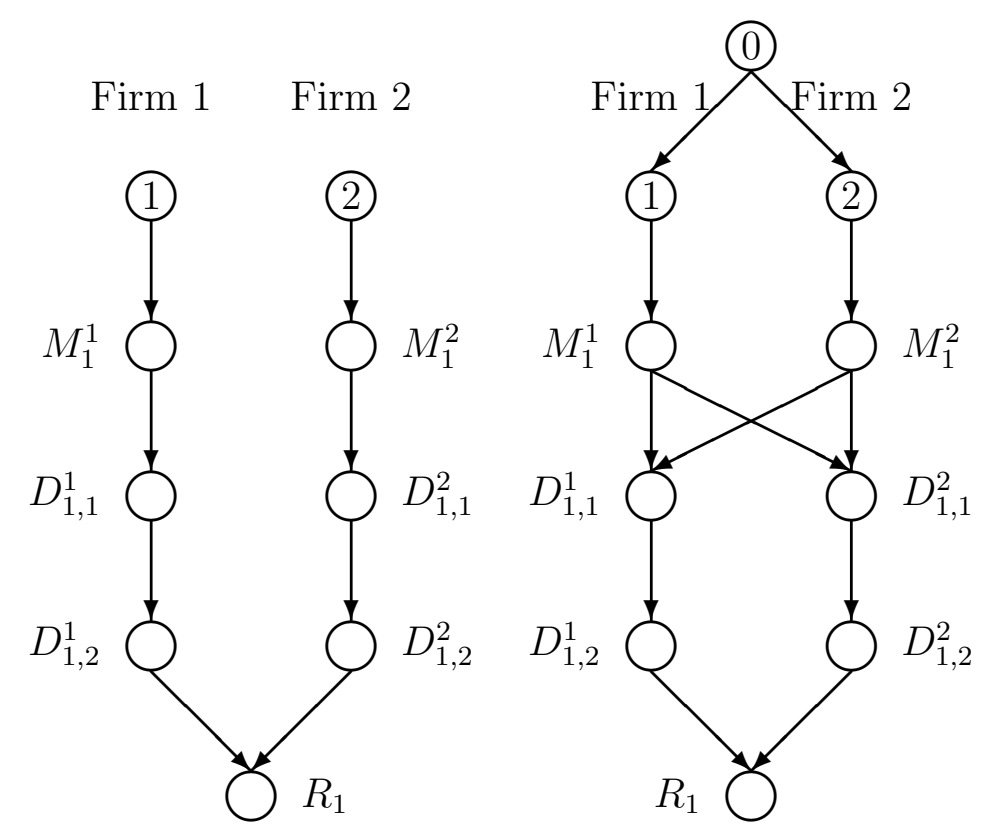

Figure 5: Merger of a Duopoly for Problem Set 1

merger Examples 1.1a through 1.1d. This problem has the variational inequality formulation given by (10b).

For simplicity, and easy reproducibility, we let all the total cost functions in the pre-merger supply chain network be equal and given by:

$$
\hat{c}_{a}\left(f_{a}\right)=f_{a}^{2}+f_{a}, \quad \forall a \in L_{i}^{0} ; i=1,2 .
$$

The demand market price at the single demand market was:

$$
\rho_{R_{1}}\left(d_{R_{1}}\right)=-d_{R_{1}}+100
$$

There were only two paths in the pre-merger network which we denote by $p_{1}$ for firm 1 and by $p_{2}$ for firm 2 . The equilibrium solution satisfying variational inequality (10b) (which can also be obtained analytically since here we have a symmetric equilibrium) was:

$$
x_{p_{1}}^{*}=x_{p_{2}}^{*}=8.72 ; \quad d_{R_{1}}^{*}=17.44 .
$$


The equilibrium demand market price was: $\rho_{R_{1}}\left(d_{R_{1}}^{*}\right)=82.56$. The total cost for both firms was: 678.20; the total revenue for both firms was: 1,440.13, and the total profit was: 761.93. The profit for each individual firm was: 380.96 .

We then considered the (complete) merger between the two firms but varied the total costs associated with the added links (cf. Figure 5) to construct the Examples 1.1a through 1.1d below, whose solutions were governed by variational inequality (17b).

\section{Example 1.1a Data and Solution}

In this example, we assumed that the total cost functions were identically equal to zero for all the new links and, of course, the original link total cost functions remained unchanged, as assumed by the merger models. Note that now there are four paths (from node 0 to node $R_{1}$ ). We let now path $p_{1}$ denote the path joining node 0 with node $R_{1}$ but following the nodes of path $p_{1}$ in the pre-merger example above; the same for $p_{2}$. Path $p_{3}$ is defined to be the path that originates in 0 and terminates in $R_{1}$ and passes through node 1 , node $M_{1}^{1}$, node $D_{1,1}^{2}$, and so on, with path $p_{4}$ being the mirror image. The optimal computed solution was:

$$
x_{p_{1}}^{*}=x_{p_{2}}^{*}=0, \quad x_{p_{3}}^{*}=x_{p_{4}}^{*}=9.67, \quad d_{R_{1}}^{*}=19.34,
$$

and the demand market price was: $\rho_{R_{1}}\left(d_{R_{1}}^{*}\right)=80.66$. The total cost was now: 619.46; the total revenue was: 1,560.47, and the total profit was: 941.01. One can see that, in this example, since the linkage costs were all zero, post-merger, it was optimal to have the volume of product produced at the original firm's manufacturing plant to be stored at the other firm's distribution center and then shipped to the demand market. As expected, and as predicted from economic theory, the profits increased.

\section{Example 1.1b Data and Solution}

Example 1.1b had the same data as Example 1.1a but now we assumed that the total cost functions on the new links (cf. Figure 5) joining each manufacturing plant with the other original firm's distribution center were no longer zero but were identical to the total cost functions on the pre-merger links (cf. (26)). The solution now was:

$$
x_{p_{1}}^{*}=x_{p_{2}}^{*}=x_{p_{3}}^{*}=x_{p_{4}}^{*}=4.36, \quad d_{R_{1}}^{*}=17.45 .
$$


The demand market price was now: $\rho_{R_{1}}\left(d_{R_{1}}^{*}\right)=83.55$. The total cost was now: 602.76 ; the total revenue was: $1,330.70$, and the total profit was: 837.94 .

Notice that now all the paths had positive flow. The profit was still higher than before the merger but not as high as when the total costs of the new links were all zero as in Example 1.1a.

\section{Example 1.1c Data and Solution}

This example was also constructed from Example 1.1a. Here, however, and in contrast to Example 1.1b, we kept the data as in Example 1.1a but now we set the total costs on the two new links joining node 0 with nodes 1 and 2 , respectively, equal to the same total cost functions as on the original links. These costs may be interpreted as the costs associated with actually merging the two firms into a single firm. We now had only two paths having positive flow. The computed solution was:

$$
x_{p_{1}}^{*}=x_{p_{2}}^{*}=0, \quad x_{p_{3}}^{*}=x_{p_{4}}^{*}=7.98, \quad d_{R_{1}}^{*}=15.96 .
$$

The demand market price was now: $\rho_{R_{1}}\left(d_{R_{1}}^{*}\right)=84.04$. The total cost was now: 573.22 ; the total revenue was: $1,341.35$, and the total profit was: 768.13 .

Interestingly, the profit associated with this merger was very close to that obtained for the duopoly (prior to the merger).

\section{Example 1.1d Data and Solution}

This example was also constructed from Example 1.1a but now all the new links had total cost functions equal to the total cost functions on the original links (cf. (26)). All paths were now again used and the symmetric solution was:

$$
x_{p_{1}}^{*}=x_{p_{2}}^{*}=x_{p_{3}}^{*}=x_{p_{4}}^{*}=3.65, \quad d_{R_{1}}^{*}=14.60 .
$$

The demand market price was now: $\rho_{R_{1}}\left(d_{R_{1}}^{*}\right)=85.40$. The total cost was now: 553.48 ; the total revenue was: $1,247.85$, and the total profit was: 694.37 . 
Table 1: Summary of Results for Set 1: Examples 1.1 (Pre-merger) and Examples 1.1a Through 1.1d (Post-merger)

\begin{tabular}{|l|c|c|c|c|c|}
\hline Measure & Ex. 1.1 & Ex. 1.1a & Ex. 1.1b & Ex. 1.1c & Ex. 1.1d \\
\hline Total Profit & 761.93 & 941.01 & 837.94 & 768.13 & 694.37 \\
\hline Total Cost & 678.20 & 619.46 & 602.76 & 573.22 & 553.48 \\
\hline Total Revenue & $1,440.13$ & $1,560.47$ & $1,440.70$ & $1,341.35$ & $1,247.85$ \\
\hline Equil. Demand & 17.44 & 19.34 & 17.45 & 15.96 & 14.60 \\
\hline Equil. Price & 82.56 & 80.66 & 83.55 & 84.04 & 85.40 \\
\hline
\end{tabular}

In this example, the profits were lower than the total profit for both firms in the duopoly (prior to the merger), even though the total costs were lower.

In Table 1, we present a summary of the results for Examples 1.1, 1.1a through 1.1d.

\section{Computational Experimentation}

In light of the above computational results, we proceeded to investigate how changes in the total cost functions associated with the top-most links joining node 0 to node 1 and node 0 to node 2 would affect profits. This question is interesting since it examines the costs associated with the merging of the two original firms. For example, these total cost functions may include risk associated with the merger, the costs associated with the integration of technology, of human resources, etc. We used the Example 1.1a data as a starting point and we set the total cost functions on the top-most links to: $\hat{c}_{a}\left(f_{a}\right)=h_{a} f_{a}$ and varied the $h_{a}$ terms. We first set these terms on both top-most links equal to 0 (as in Example 1.1a); and then to 10; to 20, and so on. Figure 6 displays the profits obtained post the merger with these costs. Note that after these terms become equal to or greater than 100, the merged firms would not produce and the profits are always zero. In fact, with further experimentation, we found that if the terms are set to 97 then zero profits occur and the firms do not produce (whereas they would still produce with both top-most links' $h_{a}$ terms equal to 96).

The above computational experimental results led us to the following theoretical result. 


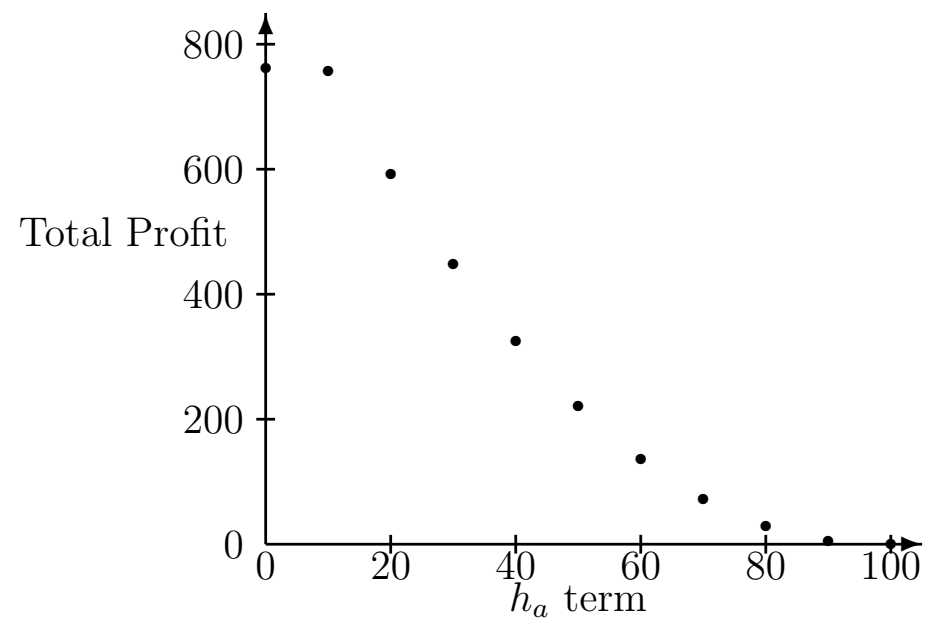

Figure 6: Total Profit vs. Merger Link Total Cost Terms: $h_{a}$ for $a=(0,1)$ and $a=(0,2)$ in Example 1.1a

\section{Theorem 4}

Consider the merger of a duopoly as depicted in Figure 5. Assume that the total link cost functions, pre-merger, are equal and given by:

$$
\hat{c}_{a}\left(f_{a}\right)=g f_{a}^{2}+k f_{a}, \quad \forall a \in L_{i}^{0} ; i=1,2,
$$

with $g, k>0$.

Assume that the demand market price function at the single demand market is:

$$
\rho_{R_{1}}\left(d_{R_{1}}\right)=-d_{R_{1}}+t
$$

with $t>0$.

Assume that the total cost functions of the merger links joining the manufacuturing nodes to the other firm's distribution nodes are zero. Assume also that the total cost functions on the top-most merger links joining node 0 to node 1 and node 0 to node 2 are equal and given by $h_{a} f_{a}=h f_{a}$ for $h>0$. Then, if

$$
\frac{t-3 k-h}{6 g+4} \leq 0,
$$


it makes no sense for the firms to merger since the profits will be zero.

Proof: The solution of this problem is governed by variational inequality (17b). We keep the definitions of the post-merger paths as above and it is clear that, post-merger, paths $p_{1}$ and $p_{2}$ will not be used and that $x_{p_{3}}^{*}=x_{p_{4}}^{*}$. We set $x_{p}=x_{p}^{*}$ for all $p \neq p_{3}$ in (17b) to obtain, after algebraic simplification, the following explicit form:

$$
\left(h+6 g x_{p_{3}}^{*}+3 k-t+2 x_{p_{3}}^{*}+2 x_{p_{3}}^{*}\right) \times\left(x_{p_{3}}-x_{p_{3}}^{*}\right) \geq 0 .
$$

Hence, if $x_{p_{3}}^{*}>0$, we must have that:

$$
x_{p_{3}}^{*}=\frac{t-3 k-h}{6 g+4}
$$

and, due to the nonnegativity constraint on the path flow(s), for $\frac{t-3 k-h}{6 q+4} \leq 0, x_{p_{3}}^{*}=0$, and the conclusion follows.

Hence, in the above computational experimentation examples, with $t=100, k=1$, and $g=1$, with $h=97$ we have that, indeed, as obtained experimentally, but now derived theoretically in Theorem 4, the profits will be zero and it makes no economic sense for such a merger. This theorem is interesting since it relates the potential success of a merger directly to the total cost functions and the demand function.

\section{Data for Examples 1.2, 1.2a - 1.2d}

Example 1.2 was, again, a pre-merger supply chain network problem, with the topology as given in Figure 5. This example, however, unlike Example 1.1, had total cost functions on the links that were not identical. The data for the total cost functions for this pre-merger, baseline example, from which the merger variants Examples 1.2a through $1.2 \mathrm{~d}$ are built, are in Table 2. The demand price function was given by (27). In Example 1.2a (as in Example 1.1a), all the new links comprising the merger had total cost functions identically equal to zero. Example 1.2b was constructed from Example 1.2a but had total cost functions on the top-most merger links equal to zero and the cost functions on the new distribution links were identical to those as in Example 1.2b. Example 1.2c, in turn, was also constructed from Example 1.2a and had zero total cost functions associated with the distribution links 
Table 2: Definition of Links and Associated Total Cost Functions for Example 1.2 - See Also Figure 5

\begin{tabular}{|c|c|c|l|}
\hline Link $a$ & From Node & To Node & Ex. 1.2: $\hat{c}_{a}\left(f_{a}\right)$ \\
\hline 1 & 1 & $M_{1}^{1}$ & $f_{1}^{2}+11 f_{1}$ \\
\hline 2 & $M_{1}^{1}$ & $D_{1,1}^{1}$ & $2 f_{2}^{2}+8 f_{2}$ \\
\hline 3 & $D_{1,1}^{1}$ & $D_{1,2}^{1}$ & $f_{3}^{2}+6 f_{3}$ \\
\hline 4 & $D_{1,2}^{1}$ & $R_{1}$ & $.5 f_{4}^{2}+5 f_{4}$ \\
\hline 5 & 2 & $M_{1}^{2}$ & $4 f_{5}^{2}+7 f_{5}$ \\
\hline 6 & $M_{1}^{2}$ & $D_{1,1}^{2}$ & $3 f_{6}^{2}+11 f_{6}$ \\
\hline 7 & $D_{1,1}^{2}$ & $D_{1,2}^{2}$ & $4 f_{7}^{2}+11 f_{7}$ \\
\hline 8 & $D_{1,2}^{2}$ & $R_{1}$ & $4 f_{8}^{2}+5 f_{8}$ \\
\hline
\end{tabular}

Table 3: Summary of Results for Set 1: Examples 1.2 (Pre-merger) and Examples 1.2a Through 1.2d (Post-merger)

\begin{tabular}{|l|c|c|c|c|c|}
\hline Measure & Ex. 1.2 & Ex. 1.2a & Ex. 1.2b & Ex. 1.2c & Ex. 1.2d \\
\hline Total Profit & 266.96 & 398.89 & 368.89 & 341.18 & 316.04 \\
\hline Total Cost & 473.94 & 540.51 & 521.18 & 482.98 & 464.69 \\
\hline Total Revenue & 740.90 & 939.40 & 840.07 & 824.16 & 780.73 \\
\hline Equil. Demand & 8.06 & 10.50 & 9.88 & 9.06 & 8.54 \\
\hline Equil. Price & 91.94 & 89.50 & 90.12 & 90.94 & 91.46 \\
\hline
\end{tabular}

and total cost functions identical to those in Example 1.1c on the top-most links emanating from node 0. Finally, Example 1.2d was constructed from Example 1.2c but had total cost functions on the new distribution links as in Example 1.2b.

We report the total profits, the total costs, and total revenues, as well as the equilibrium demands and prices for Example 1.2, and for Examples 1.2a through 1.2d, in Table 3.

Below, for completeness, we provide the computed path flow solutions for each example, along with a discussion, and results of additional computational experiments. 


\section{Example 1.2 Solution}

The paths for this example and the subsequent merger examples are as defined for Examples 1.1, 1.1a - 1.1d, since Figure 5 reflects the problem structure. The computed equilibrium path flows for this duopoly were:

$$
x_{p_{1}}^{*}=6.19, \quad x_{p_{2}}^{*}=1.87,
$$

with an equilibrium product demand of: $d_{R_{1}}^{*}=8.06$.

\section{Example 1.2a Solution}

The computed path flows were now:

$$
x_{p_{1}}^{*}=3.35, \quad x_{p_{2}}^{*}=0.00, \quad x_{p_{3}}^{*}=2.52, \quad x_{p_{4}}^{*}=4.63,
$$

with a corresponding product demand of $d_{R_{1}}^{*}=10.50$.

\section{Example 1.2b Solution}

The computed path flows were now:

$$
x_{p_{1}}^{*}=3.82, \quad x_{p_{2}}^{*}=0.00, \quad x_{p_{3}}^{*}=2.23, \quad x_{p_{4}}^{*}=3.83,
$$

with $d_{R_{1}}^{*}=9.88$.

\section{Example 1.2c Solution}

The computed path flows were now:

$$
x_{p_{1}}^{*}=2.70, \quad x_{p_{2}}^{*}=0.00, \quad x_{p_{3}}^{*}=2.15, \quad x_{p_{4}}^{*}=4.21,
$$

with $d_{R_{1}}^{*}=9.06$.

\section{Example 1.2d Solution}

The computed path flows were now:

$$
x_{p_{1}}^{*}=3.06, \quad x_{p_{2}}^{*}=0.00, \quad x_{p_{3}}^{*}=1.89, \quad x_{p_{4}}^{*}=3.58,
$$




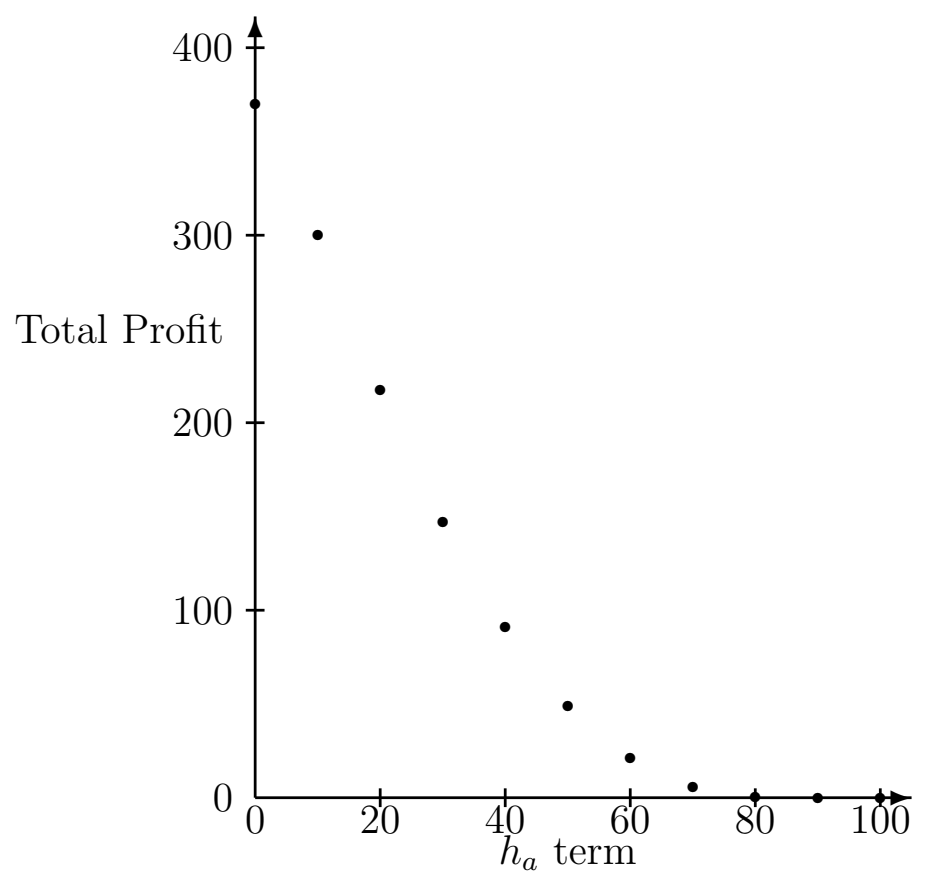

Figure 7: Total Profit vs. Merger Link Total Cost Terms: $h_{a}$ for $a=(0,1)$ and $a=(0,2)$ in Example 1.2a

with a corresponding product demand of $d_{R_{1}}^{*}=8.54$.

It is interesting to see that in all the Examples 1.2a through $1.2 \mathrm{~d}$, path $p_{2}$ never had positive product flow. This can be expected, given that the storage cost on the storage link on that path is substantially higher that on the corresponding link comprising path $p_{1}$; moreover, the manufacturing link on that path has a higher total cost function than the other manufacturing link.

\section{Additional Computational Experimentation}

We continued to explore the impact of the values of the total cost functions on the topmost merger links, as we had following Example 1.1d above. We conducted similar experiments. We used Example 1.2a but added total costs to the top-most links given by $\hat{c}_{a}\left(f_{a}\right)=h_{a} f_{a}$. The plot of the profits vs. the assigned $h_{a}$ values is given in Figure 7 . When $h_{a}=80$ the profits were reduced to .23. From $h_{a}=90$ and higher, the profits were always equal to zero and, clearly, zero volume of the product was produced. Obviously, if the associated such merger total cost functions are such, then it makes no economic sense for the firms in this duopoly to merge. 
The Firms
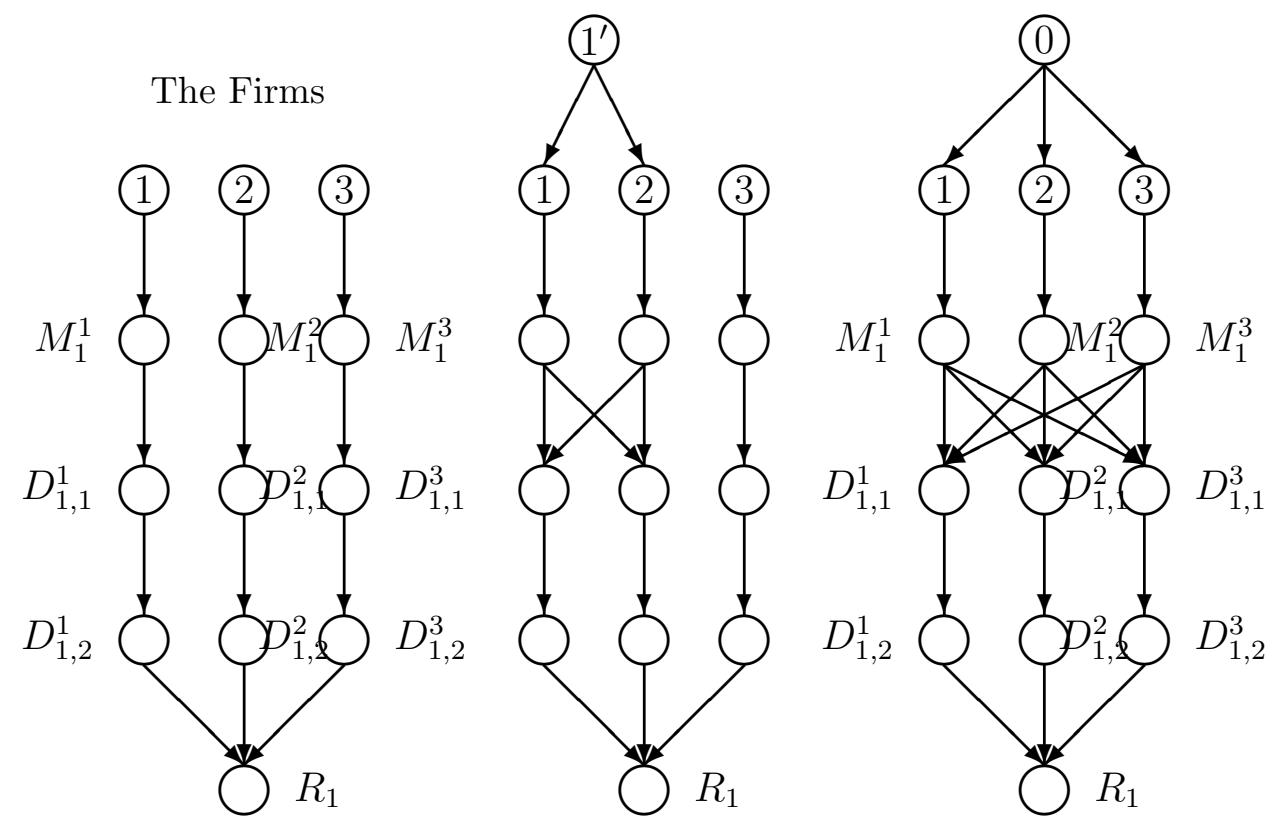

Figure 9: Supply Chain Network Structure of the Three Firms Prior to and Post the Partial and Full Mergers in Problem Set 2

\subsection{Set 2}

In this Subsection, we considered the solution of oligopolies and their partial and full mergers where the pre-merger number of competing firms in the oligopoly was three. Please refer to Figure 8 for the structure of the supply chain networks pre-merger and post-merger (partial and complete).

Example 2.1 serves as the baseline (Case 0) for the examples in this set: Examples 2.1a through 2.1h. Examples 2.1a through 2.1d correspond to the partial merger where firms 1 and 2 merge and then compete with the original firm 3. Examples 2.1e through 2.1h correspond to the complete merger among all three original firms. The relevant measures are reported in Table 4 for the partial merger examples and in Table 5 for the complete merger.

\section{Example 2.1 Data and Solution}

This pre-merger example assumed that the total cost functions on all links of the pre-merger 
supply chain network in Figure 8 were as in (26). The total profit for this oligopoly was 960.38, with each firm earning one third, that is, 320.12 in profit. The computed equilibrium path flows on the three paths, with each corresponding to a particular firm in the oligopoly was: 8.00 .

\section{Examples 2.1a Through 2.1d (Partial Merger) Data and Solutions}

Examples 2.1a through 2.1d were constructed from Example 2.1 but, respectively, had the total links cost functions varied on the merger links as in Example 1.1a through 1.1d, respectively. The results are summarized in Table 4.

In Example 2.1a, the profit of the original firm 3, which did not merge with the first two original firms, was: 309.55. The profit of the merged firm was: 791.66. In Example 2.1b, the profit of the unmerged firm was: 320.00, which is essentially the same profit obtained by each firm in the original oligopoly prior to any merger. The profit of the new, merged firm was now: 704.15. In Example 2.1c, the third original firm's profit was now 331.32, and the profit of the newly merged firm was: 643.32. In Example 2.1d, the original third firm's profit was now 333.40, which is greater than it was for each of the three firms in the original oligopoly. The total profit of the firms in the merger was: 587.03.

One can see that, in each of the examples, Example 2.1a through 2.1c, the merged firm had higher total profit than the sum of those two firms' profits in the oligopoly (pre-merger). This was not the case, however, for Example 2.1d in which each of the newly merged firms had, in effect, a profit of 293.51, which is lower than its original 320.12. It is also worth noting that, in the case of Example 2.1c, all the firms gained from the partial merger. Recall that in this merger example there were zero costs associated with the new distribution links. In Example 2.1b, both merged firms gained and the third unmerged firm was hardly affected in terms of change in its profits.

It is also important to note that the total costs were lower in all these partial post-merger examples, but the revenues were higher only in Examples 2.1a and 2.1b.

Interestingly, as emphasized by Creane and Davidson (2004), the traditional approach to modeling mergers has generated a number of "intuitively unappealing results." For example, 
Table 4: Summary of Results for Set 2: Examples 2.1 (Pre-merger) and Examples 2.1a Through 2.1d (Partial Merger)

\begin{tabular}{|l|c|c|c|c|c|}
\hline Measure & Ex. 2.1 & Ex. 2.1a & Ex. 2.1b & Ex. 2.1c & Ex. 2.1d \\
\hline Total Profit & 960.38 & $1,101.21$ & $1,024.15$ & 974.64 & 920.43 \\
\hline Total Cost & 863.10 & 804.08 & 799.64 & 782.77 & 773.26 \\
\hline Total Revenue & $1,823.48$ & $1,905.29$ & $1,823.78$ & $1,757.41$ & $1,693.69$ \\
\hline Equil. Demand & 24.00 & 25.62 & 24.00 & 22.75 & 22.61 \\
\hline Equil. Price & 76.00 & 74.38 & 76.00 & 77.25 & 77.39 \\
\hline
\end{tabular}

the authors note that in quantity-setting games, as is the case in our pre-merger and postmerger models, "mergers typically are not profitable for insiders, but are profitable for nonmerging firms (outsiders)." We see that in our framework that need not be the case. Indeed, in all the partial merger examples, Examples 2.1a through 2.1c, the "insiders" all gained from the merger and the "outsider" does not gain until Example 2.1c. Hence, we see that our supply chain network perspective is both intuitively appealing and computationally tractable. Here, through the above examples, we show that, in contrast to the quote from Pepall, Richard, and Norman (1999) in Section 1, that we have constructed a simple economic model in which there are sizable profitability gains for the firms participating in a horizontal merger that is not a merger to a monopoly. Furthermore, we see from the additional computational experiments in Section 3.1 above, that whether or not the merger of firms in an oligopoly is profitable depends upon the total costs associated with joining/merging the firms. The above examples, hence, help to clarify the "merger paradox." Importantly, one must, in advance, analyze carefully the total costs and revenues that can be expected from any proposed merger and take all the firms' economic activities into consideration, ideally, from a network perspective.

\section{Examples 2.1e Through 2.1h (Complete Merger) Data and Solutions}

These examples correspond to the complete merger of the three firms. Examples 2.1e has total costs on the new links as discussed in Example 2.1a. Example 2.1f followed the formalism in Example 2.1b, and so on, until Example 2.1h, in which all the links post-merger had identical total cost functions to the pre-merger functions in Example 2.1d. 
Table 5: Summary of Results for Set 2: Examples 2.1 (Pre-merger) and Examples 2.1e Through 2.1h (Complete Merger)

\begin{tabular}{|l|c|c|c|c|c|}
\hline Measure & Ex. 2.1 & Ex. 2.1e & Ex. 2.1f & Ex. 2.1g & Ex. 2.1h \\
\hline Total Profit & 960.38 & $1,176.41$ & $1,091.32$ & 987.76 & 923.14 \\
\hline Total Cost & 863.10 & 658.91 & 665.40 & 644.63 & 642.45 \\
\hline Total Revenue & $1,823.48$ & $1,835.32$ & $1,756.72$ & $1,632.39$ & $1,565.60$ \\
\hline Equil. Demand & 24.00 & 24.22 & 22.74 & 20.54 & 19.43 \\
\hline Equil. Price & 76.00 & 75.78 & 77.26 & 79.46 & 80.57 \\
\hline
\end{tabular}

The results are reported in Table 5. It is interesting to compare the "per original firm" profit that results from the complete merger. In the case of Example 2.1e, the profit divided equally by three yields: 392.13, in the case of Example 2.1f, this value is: 363.77. In the case of Example 2.1g, the per original firm profit is now: 329.25, and, finally. In the case of Example 2.1h, this value is: 307.71. Hence, only in the case of Example 2.1h does the complete merger result in a "per firm" profit that is less than that obtained for the original oligopoly, the value of which (cf. Example 2.1 above) was: 320.12. It is also very interesting to note that in the case of Example $2.1 \mathrm{~g}$ and its partial merger counterpart, Example 2.1c, that the original third firm, which did not participate in the partial merger actually had higher profits than when it did participate in the merger.

\subsection{Problem Set 3}

In this Subsection, we continued our computational/numerical experiments. In this set, we solved pre- and post-merger problems consisting of four firms initially.

\section{Example 3.1 Data and Solution}

We proceeded to investigate the case of two firms in a four firm oligopoly merging. Recall that, according to Salant, Switzer, and Reynolds (1983), in a Cournot oligopoly, it is not usually advantageous for quantity-setting firms to merge unless almost all of them merge. In investigating the merger of two firms out of four, we clearly do not have the majority merging. 
In this example, which was an oligopoly with four firms, all faced the same total cost functions on the links as in Example 2.1. Essentially, we added another firm to the oligopoly in Problem 2.1, assumed the same total cost functions on its links as were on all the other firms' links, and kept the same demand price function.

Each firm in this oligopoly produced 7.38 units of the product. Hence, the demand for the product was: 29.52 and the demand price was: 70.48 . The revenue for all the firms was: 2, 080.95; the total costs were: 989.76 and the profit for all firms together was: 1,091.20, which means that each firm realized a profit of 272.80. As predicted by economic theory, since there was more competition than when there were only three firms in the oligopoly, the profit for each decreased. Recall that in the three-firm case, Example 2.1 above, each firmed earned a profit of 320.12 .

\section{Example 3.1a Data and Solution}

This example had the same data as Example 3.1 but now we considered the partial merger with the first two firms in Example 3.1 merging. We assumed that there were zero total costs associated with all the new links. Each unmerged firm produced 7.23 units of the product; whereas only two paths of the new firm had positive flow and the flow on each such path was equal to: 8.23. The demand was now: 30.92 and the demand market price was: 69.08. The total cost was now: 932.21; the total revenue was: 2, 136.30, and the profit was: 1,204.09. Each of the two firms that did not enter into the merger had a profit of 261.30, whereas each of the two firms that did merge had a profit of 670.00 . Hence, after this partial merger, the merging firms, that is, the "insiders" gained substantially.

\section{Example 3.2 Data and Solution}

In this example, we, again considered four firms competing in an oligopolistic manner. The total cost functions on each of the links was, again, given by:

$$
\hat{c}_{a}=f_{a}^{2}+f_{a}, \quad \forall a \in L^{0}
$$

However, rather than a single demand market, we now had two demand markets. The demand price function associated with the first demand market was as in (27). The demand 
price function for the second demand market was:

$$
\rho_{R_{2}}\left(d_{R_{2}}\right)=-d_{R_{2}}+200
$$

The Cournot-Nash equilibrium solution consisted of each firm in the oligopoly supplying demand market $R_{1}$ an amount .55 of the product and each supplying demand market $R_{2}$ an amount: 14.81 of the product. The cost was: 3,959.15, the revenue was: 8,556.96, and the profit, hence, was: 4,597.81 with each firm earning a profit of: 1, 149.45. The demand market price at $R_{1}$ was: 97.32 and at $R_{2}: 140.75$.

\section{Example 3.2a Data and Solution}

Example 3.2a was a partial merger problem. We assumed that the first two firms only in Example 3.2 merged. Also, we assumed that the new links associated with the merger (the top-most links and the added distribution links between the first two original firms) had total cost functions that were all equal to zero. The total cost was now: 3,954.74, the revenue was: $9,114.79$, and the profit was: 5,160.05. Interestingly, only the newly merged firm supplied demand market $R_{1}$ at an amount of 7.25 , whereas the demand at $R_{2}$ was now: 60.53. The profit of each of the two unmerged firms was now: $1,102.50$ whereas the profit of each merged firm was: 1,567.27, a value significantly higher than pre-merger.

Again, we have constructed a relatively simple example for which the "insiders" in the merger gain. We were able to accomplish this through the powerful tool of computational methods and numerical experimentation.

\section{Example 3.2b Data and Solution}

In our final example, we explored the effects of non-zero total cost functions on all the merger links (as was done previously above in Examples 1.2d. 2.1d, and 2.1h). Example 3.2b had the same data as Example 3.2a except that all the new links had total link cost functions:

$$
\hat{c}_{a}\left(f_{a}\right)=f_{a}^{2}+f_{a}, \quad \forall a \in L^{2} .
$$

The total cost was now: 3, 677.09, the revenue was: $8,184.99$, and the profit was: 4,507.90, which was lower than the profit pre-merger. The demand at $R_{1}$ was: 1.70 , with a demand 
price of 98.30. The demand at $R_{2}$ was: 55.48 at a demand price of: 144.52 . The profit of each of the two unmerged firms was now: 1,207.69 whereas the profit of each merged firm was: $1,046.26$. Hence, now the unmerged firms would realize a higher individual profit than pre-merger.

\section{Summary and Conclusions}

In this paper, we developed a supply chain network framework in an oligopolistic setting, which allows one to explore partial and full mergers between/among the competing firms. We formulated both the pre and the post merger problems as variational inequality problems and then proposed an algorithm, which fully exploits the underlying structure of these network problem and yields closed form expressions at each iterative step. We then conducted extensive computational experiments in order to explore issues surrounding what has been termed in the literature as the "merger paradox."

We believe that the richness of the network formalism proposed here for the investigation of economic issues surrounding mergers yields insights into mergers and the associated costs, revenues, and profits. Furthermore, the network structure allows one to investigate and visualize the structure of the problems both pre and post the particular mergers and to assign costs as relevant. The models, we believe, are intuitively appealing. In addition, this paper vividly illustrates the power of computational methodologies to explore issues regarding competing firms and possible mergers.

Moreover, this research can be extended in numerous directions. One can construct not only single mergers, but multiple mergers between/among subsets of firms; one can construct multiproduct versions of the models developed here, and one can also consider international issues concerning mergers and acquisitions.

\section{Acknowledgments}

The author thanks the three anonymous reviewers and the Editor-in-Chief, Professor Berc Rustem, for many helpful suggestions that improved the presentation of this work and also led to additional theoretical results.

This research was supported by the John F. Smith Memorial Fund at the Isenberg School 
of Management. This support is gratefully acknowledged.

\section{References}

Bazaraa, M. S., Sherali, H. D., Shetty, C. M., 1993. Nonlinear Programming: Theory and Algorithms. John Wiley \& Sons, New York.

Beverage World, 2007. Molson Coors Brewing Co. http://www.beverageworld.com/content/view/33296/

Brown, G., Keegan, J., Vigus, B., Wood, K., 2001. The Kellogg company optimizes production, inventory and distribution. Interfaces 31, 1-15.

CNNMoney.com, 1999. Exxon-Mobil merger done. November 30.

Cournot, A. A., 1838. Researches into the Mathematical Principles of the Theory of Wealth, English translation, MacMillan, London, England, 1897.

Creane, A., Davidson, C., 2004. Multidivisional firms, internal competition and the merger paradox. Canadian Journal of Economics 37, 951-977.

Dafermos, S., Nagurney, A. 1987. Oligopolistic and competitive behavior of spatially separated markets. Regional Science and Urban Economics 17, 245-254.

Dafermos, S., Sparrow, F. T., 1969. The traffic assignment problem for a general network. Journal of Research of the National Bureau of Standards 73B, 91-118.

Dupuis, P., Nagurney, A., 1993. Dynamical systems and variational inequalities. Annals of Operations Research 44, 9-42.

Farrell, J., Shapiro, C., 1990. Horizontal mergers: An equilibrium analysis. American Economic Review 80, 107-126.

Flam, S. P., Ben-Israel, A., 1990. A continuous approach to oligopolistic market equilibrium. Operations Research 38, 1045-1051. 
Friedman, J., 1982. Oligopoly theory. In: Handbook of Mathematical Economics, vol. II, Arrow, K. J., Intriligator, M. D. (Eds), North Holland, Amsterdam, The Netherlands, pp. $490-534$, .

Gabay, D., Moulin, H., 1980. On the uniqueness and stability of Nash equilibria in noncooperative games, in Applied Stochastic Control of Econometrics and Management Science, A. Bensoussan, P. Kleindorfer, and C. S. Tapiero, editors, North-Holland, Amsterdam, The Netherlands.

Ghosal, V., Stennek, J., Editors, 2007. The Political Economy of Antitrust, Elsevier, Amsterdam, The Netherlands.

Global News Wire, 2008. Delta and Northwest merge, creating premier global airline. Press release, October 29.

Gupta, D., Gerchak, Y., 2002. Quantifying operational synergies in a merger and acquisition. Management Science 48, 517-534.

Hakkinen, L., Norrman, A., Hilmola, O.-P., Ojala, L., 2004. Logistics integration in horizontal mergers and acquisitions. The International Journal of Logistics Management 15, $27-42$.

Herd, T., Saksena, A. K., Steger, T. W., 2005. Delivering merger synergy: A supply chain perspective on achieving high performance. Outlook - Point of View, Accenture, May.

Kusstatscher, V., Cooper, C. L., 2005. Managing Emotions in Mergers and Acquisitions. Edward Elgar Publishing, Cheltenham, England.

Langabeer, J., Seifert, D., 2003. Supply chain integration: The key to merger success (synergy). Supply Chain Management Review 7, 58-64.

Meschi, M., 1997. Analytical perspectives on mergers and acquisitions. A survey. Paper Number 5-97, ISSN number 1366-6290, Centre for International Business Studies, South Bank University, London, England. 
Min, H., Zhou, G., 2002. Supply chain modeling: past, present, future. Computers and Industrial Engineering 43, 231-249.

Murphy, F. H., Sherali, H. D., Soyster, A. L., 1982. A mathematical programming approach for determining oligopolistic market equilibrium. Mathematical Programming 24, 92-106.

Nagurney, A., 1993. Network Economics: A Variational Inequality Approach. Kluwer Academic Publishers, Dordrecht, The Netherlands.

Nagurney, A. 2006a. On the relationship between supply chain and transportation network equilibria: A supernetwork equivalence with computations. Transportation Research E 42, 293-316.

Nagurney, A. 2006b. Supply Chain Network Economics: Dynamics of Prices, Flows and Profits. Edward Elgar Publishing, Cheltenham, England.

Nagurney, A., 2009. A system-optimization perspective for supply chain network integration: The horizontal merger case. Transportation Research E 45, 1-15.

Nagurney, A., Dong, J., Zhang, D., 2002. A supply chain network equilibrium model. Transportation Research E 38, 281-303.

Nagurney, A., Dupuis, P., Zhang, D., 1994, A dynamical systems approach for network oligopolies and variational inequalities. Annals of Operations Research 28, 263-293.

Nagurney, A., Takayama, T., Zhang, D., 1995. Massively parallel computation of spatial price equilibrium problems as dynamical systems. Journal of Economic Dynamics and Control 18, 3-37.

Nagurney, A., Zhang, D., 1996a. Stability of spatial price equilibrium modeled as a projected dynamical system. Journal of Economic Dynamics and Control 20, 43-63.

Nagurney, A., Zhang, D., 1996b. Projected Dynamical Systems and Variational Inequalities with Applications, Kluwer Academic Publishers, Boston, Massachusetts.

Nash, J. F., 1950. Equilibrium points in n-person games. Proceedings of the National 
Academy of Sciences, USA 36, 48-49.

Nash, J. F., 1951. Noncooperative games. Annals of Mathematics 54, 286-298.

Pepall, L., Richards, D., Norman, G., 1999. Industrial Organization: Contemporary Theory and Practice. South-Western College Publishing, Cleveland, Ohio.

Perry, M. K., Porter, R. H., 1985. Oligopoly and the incentive for horizontal merger. American Economic Review 75, 219-227.

Phoenix Business Journal, 2009. Wells Fargo, Wachovia complete merger. January 2.

Salant, S., Switzer, S., Reynolds, R., 1983. Losses due to merger: The effects of an exogenous change in industry structure on Cournot-Nash equilibrium. Quarterly Journal of Economics 48, 185-200.

Sandholm, W. H., Dokumaci, E., Lahkar, R., 2008. The projection dynamic and the replicator dynamic. Games and Economic Behavior 64, 666-683.

Soylu, A., Oruc, C., Turkay, M., Fujita, K., Asakura, T., 2006. Synergy analysis of collaborative supply chain management in energy systems using multi-period MILP. European Journal of Operational Research 174, 387-403.

Tirole, J., 1988. The Theory of Industrial Organization. MIT Press, Cambridge, MA.

TradingMarkets.com, 2008. Anheuser-Busch shareholders okays InBev merger. November 12.

Xu, S., 2007. Supply Chain Synergy in Mergers and Acquisitions: Strategies, Models and Key Factors, PhD dissertation, University of Massachusetts, Amherst, Massachusetts.

Zhang, D., 2006. A network economic model for supply chain vs. supply chain competition. Omega 34, 283-295.

Zhang, D., Dong, J., Nagurney, A., 2003. A supply chain network economy: modeling and qualitative analysis. In: Nagurney, A. (Ed), Innovations in Financial and Economic 
Networks. Edward Elgar Publishing, Cheltenham, England, pp. 197-213.

Zhang, D., Nagurney, A., 1997. Formulation, stability, and computation of traffic network equilibria as projected dynamical systems. Journal of Optimization Theory and its Applications 93, 417-444. 


\section{Appendix}

For convenience of the reader, we recall the Euler method, which was applied to compute the solutions to the numerical examples in Section 3. The Euler method is a special case of the general iterative scheme proposed by Dupuis and Nagurney (1993) for the determination of stationary points of projected dynamical systems; equivalently, solutions of variational inequality problems. In the context of our models, the Euler method resolves the network problems into subproblems that are solved, at each iteration, explicitly and in closed form. Below, we provide these explicit formulae. A variety of economic equilibrium problems (and the associated tatonnement processes) have been modeled to-date as projected dynamical systems; see Nagurney, Takayama, and Zhang (1995) and Nagurney and Zhang (1996a) for such models of dynamic spatial price models; see Nagurney, Dupuis, and Zhang (1994) for dynamic oligopolies modeled and solved as projected dynamical systems; see Zhang and Nagurney (1997) and Nagurney and Zhang (1996b) for projected dynamical systems models of transportation network equilibria. In the context of additional economic and game theoretic problems, Sandholm, Dokumaci, and Lahkar (2008) explore the relationships between the projection dynamic of Dupuis and Nagurney (1993) (see also Nagurney and Zhang (1996b)) and the replicator dynamic.

Specifically, recall that at an iteration $\tau$ of the Euler method (see also Nagurney and Zhang (1996b)) one must compute:

$$
X^{\tau+1}=P_{\mathcal{K}}\left(X^{\tau}-a_{\tau} F\left(X^{\tau}\right)\right),
$$

where $P_{\mathcal{K}}$ is the projection on the feasible set $\mathcal{K}$ and $F$ is the function that enters the variational inequality problem: determine $X^{*} \in \mathcal{K}$ such that

$$
\left\langle F\left(X^{*}\right)^{T}, X-X^{*}\right\rangle \geq 0, \quad \forall X \in \mathcal{K},
$$

where $\langle\cdot, \cdot\rangle$ is the inner product in $n$-dimensional Euclidean space, $X \in R^{n}$, and $F(X)$ is an $n$-dimensional function from $\mathcal{K}$ to $R^{n}$, with $F(X)$ being continuous.

Clearly, all the variational inequality problems (10a), (10b), and (17a), (17b), and (25a), (25b) can be put into the above standard form (A.2). As shown in Dupuis and Nagurney (1993); see also Nagurney and Zhang (1996b), for convergence of the general iterative scheme, 
which induces the Euler method, among other methods, the sequence $\left\{a_{\tau}\right\}$ must satisfy: $\sum_{\tau=0}^{\infty} a_{\tau}=\infty, a_{\tau}>0, a_{\tau} \rightarrow 0$, as $a_{\tau} \rightarrow \infty$. Specific conditions for convergence of this scheme to the solution of variational inequality (A.2) can be found for a variety of networkbased problems, similar to those constructed here, in Nagurney and Zhang (1996b) and the references therein.

\section{Explicit Formulae for the Euler Method Applied to the Pre-Merger Supply Chain} Network Variational Inequality (10b)

The elegance of this procedure for the computation of solutions to the supply chain network problems modeled in Section 2 can be seen in the following explicit formulae. Indeed, (A.1) for the supply chain network pre-merger variational inequality problem (10b) yields the following:

$x_{p}^{\tau+1}=\max \left\{0, x_{p}^{\tau}+a_{\tau}\left(\rho_{R_{k}}\left(\sum_{p \in P_{R_{k}}^{0}} x_{p}^{\tau}\right)-\frac{\partial \rho_{k}\left(\sum_{p \in P_{R_{k}}^{0}} x_{p}^{\tau}\right)}{\partial d_{R_{k}}} \sum_{p \in P_{R_{k}^{i}}^{0}} x_{p}^{\tau}-\frac{\partial \hat{C}_{p}\left(x^{\tau}\right)}{\partial x_{p}}\right)\right\}, \quad \forall i, \forall k, \forall p \in P_{R_{k}^{i}}^{0}$.

\section{Explicit Formulae for the Euler Method Applied to the Complete Post-Merger Supply Chain Network Variational Inequality (17b)}

In the case of the complete post-merger problem developed in Section 2.2, the iterative step (A.1) for the supply chain network post-merger variational inequality problem (17b) yields the following:

$x_{p}^{\tau+1}=\max \left\{0, x_{p}^{\tau}+a_{\tau}\left(\rho_{R_{k}}\left(\sum_{p \in P_{R_{k}}^{1}} x_{p}^{\tau}\right)-\frac{\partial \rho_{k}\left(\sum_{p \in P_{R_{k}}^{1}} x_{p}^{\tau}\right)}{\partial d_{R_{k}}} \sum_{p \in P_{R_{k}}^{1}} x_{p}^{\tau}-\frac{\partial \hat{C}_{p}\left(x^{\tau}\right)}{\partial x_{p}}\right)\right\}, \quad \forall k, \forall p \in P_{R_{k}}^{1}$.

Explicit Formulae for the Euler Method Applied to the Partial Post-Merger Supply Chain Network Variational Inequality (25b)

The iterative step (A.1), in the case the supply chain network partial post-merger variational 
inequality problem (25b), yields the following expressions:

$x_{p}^{\tau+1}=\max \left\{0, x_{p}^{\tau}+a_{\tau}\left(\rho_{R_{k}}\left(\sum_{p \in P_{R_{k}}^{2}} x_{p}^{\tau}\right)-\frac{\partial \rho_{k}\left(\sum_{p \in P_{R_{k}}^{2}} x_{p}^{\tau}\right)}{\partial d_{R_{k}}} \sum_{p \in P_{R_{k}^{i}}^{2}} x_{p}^{\tau}-\frac{\partial \hat{C}_{p}\left(x^{\tau}\right)}{\partial x_{p}}\right)\right\}, \quad \forall i, \forall k, \forall p \in P_{R_{k}^{i}}^{2}$.

We have utilized the variational inequality problems (10b), (17b), and (25b) to solve since, due to the simplicity of the corresponding feasible sets, the respective realizations of the Euler method allow for the above explicit formulae. 